\title{
Coupling the Permeability and Permittivity of Space to the Electron Orbital Time: Potential Phase Shift Between Entanglement Latency and the Universe's Final Epoch
}

\author{
Michael Persinger*, Trevor Carniello \\ Biophysics Laboratory, Biomolecular Sciences Program, Laurentian University, Sudbury, Canada
}

Email address:

mpersinger@laurentian.ca (M. Persinger)

${ }^{*}$ Corresponding author

To cite this article:

Michael Persinger, Trevor Carniello. Coupling the Permeability and Permittivity of Space to the Electron Orbital Time: Potential Phase Shift Between Entanglement Latency and the Universe's Final Epoch. International Journal of Astrophysics and Space Science.

Vol. 5, No. 1, 2017, pp. 1-5. doi: 10.11648/j.jjass.20170501.11

Received: November 30, 2016; Accepted: December 10, 2016; Published: February 13, 2017

\begin{abstract}
The strength of the magnetic field for different ratios of matter densities relative to the permittivity of a vacuum solves for values approaching the velocity of light. When the strength of the field associated with densities similar to liquid water, ice, or stars (such as the Sun) is considered with respect to the magnetic permeability and average mass density of the universe, the emergent velocity is $\sim 10^{23} \mathrm{~m} \cdot \mathrm{s}^{-1}$. This value has been derived from several approaches as the latency for excess correlation or "entanglement" and is consistent with a process that might explain the integrity of large-scale spatial structure over distances that are within fractions of the universe's present diameter. The estimated latency to traverse this diameter with this velocity relative to the total duration of the universe (the final epoch) when considered as an Aharanov-Bohm type phase shift, results in an energy quantum that is convergent with Planck's constant. One interpretation is that the duration of a single electron's orbit is the phase shift between duration (latency) to traverse the universe and its total duration (final epoch). If this approach is valid then non-local effects and related excess correlations (Schrödinger's "entanglement") between photon emissions and specific dynamics of densities similar to liquid water may be a property of these conditions immersed within an average universal mass density of about one proton per cubic meter. It may also accommodate the challenges of understanding the apparent homogeneity across large scale space.
\end{abstract}

Keywords: Excess Correlation, Schrödinger's “Entanglement”, Permittivity \& permeability, Mass Density, Large Scale Structures, Eddington's Number, Aharanov-Bohm, Planck's Constant

\section{Introduction}

Although a central aspiration of physics and cosmology is to distillate all complexities to a single unified equation, the quintessential solution may instead reflect convergence and congruence between quantitative values derived from markedly different assumptions and perspectives. One of the advantages of predicting properties at the largest (upper boundary) or smallest (lower boundary) spatial or temporal unit of a phenomenon is that it assumes no intrinsic variability that could contribute stochastic or unknown sources of variance to the quantitative descriptions. When the universe is considered as a set at its maximum value for duration (the final epoch) and space $[1,2]$, its large structure characteristics [3, 4] should dominate the constants and properties of space that are observed or inferred within local, causal phenomena. At the level of the total set, the processes that interconnect space and may allow non-locality may be more easily discerned.

If mass density $\sigma$, magnetic field strength $B$ and the electric field medium $\varepsilon_{0}$ or $8.85 \cdot 10^{-12} \mathrm{~A}^{2} \cdot \mathrm{s}^{4} \cdot \mathrm{kg}^{-1} \cdot \mathrm{m}^{-3}$ are representative of the general properties of space then their quantitative relationships and the deductions derived from these values should be consistent across levels of discourse. The relationship:

$$
\mathrm{B}^{2}=\sigma \cdot \varepsilon_{\mathrm{o}}^{-1}
$$

when $\sigma=$ one proton per $\mathrm{m}^{3}\left(1.67 \cdot 10^{-27} \mathrm{~kg}\right)$ produces a 
magnetic field strength of $\sim 13.7 \mathrm{nT}$. This particular density converges with Eddington's Number for the estimated mass of the universe assuming one unit is the mass of a proton. This total mass is also within the same order of magnitude as that estimated empirically by the averages of $\sim 10^{11}$ stars per galaxy and $\sim 10^{11}$ galaxies within the universe assuming the average for each stellar mass is that of the sun $\left(\sim 2 \cdot 10^{30} \mathrm{~kg}\right)$. If the density range was between 0.5 to 2 times the unit value the range would be still be between 11 to $15 \mathrm{nT}$ [5].

This central value is within measurement error of Fischbach's geomagnetic limit for the rest mass of a photon, inferred from satellite measurements of the earth's magnetic field. It was $11.8 \mathrm{nT}$ [6]. The resulting estimate of the upper limit of the photon mass was $<10^{-51} \mathrm{~kg}$ which is the same order of magnitude calculated from the much larger Jovian magnetic field by Davis et al [7] Both of these values are within less than one order of magnitude of $<2 \cdot 10^{-52} \mathrm{~kg}$ estimate by Tu et al [8].

The equivalent transformation for the equation (1) within a much more dense medium, such as water $\left(10^{3} \mathrm{~kg} \cdot \mathrm{m}^{-3}\right)$ results in a magnetic field flux density of $\sim 1 \cdot 10^{7} \mathrm{~T}$. Applied to the magnetic moment of a proton $\left(1.4 \cdot 10^{-26} \mathrm{~A} \cdot \mathrm{m}^{2}\right)$ the resulting energy is $1.5 \cdot 10^{-19} \mathrm{~J}$ or within the range of the near infrared domain of visible light. Specifically, when divided by Planck's constant $\left(6.626 \cdot 10^{-34} \mathrm{~J} \cdot \mathrm{s}\right)$ the frequency is $0.23 \cdot 10^{15}$ $\mathrm{Hz}$. Assuming the value for the velocity of light in a vacuum, the equivalent wavelength is $\sim 1.30 \mu \mathrm{m}$.

This value is pivotal. Bohr's remarkable insight [9] while relating quantum properties of an electron and proton indicated that the frequency and magnitude of the quantum of energy involved if one proton is removed from another could be described as:

$$
v_{\mathrm{r}}=1.32 \omega_{\mathrm{o}} \sqrt{ }\left(\mathrm{m} \cdot \mathrm{M}^{-1}\right)
$$

where $\omega_{\mathrm{o}}$ was $6.2 \cdot 10^{15} \mathrm{~Hz}$ and $\mathrm{m}$ and $\mathrm{M}$ were the mass of the electron and proton, respectively. The resulting $v_{\mathrm{r}}$ from this relation was $1.57 \mu$. If the constant was not included the predicted value $(\sim 1.30 \mu \mathrm{m})$ would be within the median between these two boundaries. Realizing that the value for the energy that resulted in the specific value of this wavelength was derived from a magnetic field strength from a discrete mass assumption for density, it is possible that the convergence between Bohr's equation (2) and the resulting energy from the universal background could be more exact if empirical measurements of the parameters were obtained.

The movement or the removing of one proton from another can be considered an operation that is similar to the dynamics of the hydronium ion $\mathrm{H}_{3} \mathrm{O}^{+}$. For this configuration the extra proton is sequestered within a single water molecule for $\sim 10^{-12} \mathrm{~s}$ before it is displaced to another water molecule [10]. The Grotthuss chain or saltatory sequence is the most frequently employed metaphors for the description of this movement. As shown by Persinger [11], when this specific timing $\left(\sim 10^{-12} \mathrm{~s}\right)$ is multiplied by the ratio of the magnetic moment of the proton relative to its unit charge the resulting area produces a linear distance of $0.297 \mathrm{~nm}$ or the distance between water molecules. In other words the duration of the hydronium ion could be intrinsically coupled to the diffusivity of the dynamics of water.

The optimal cross-sectional area through which the process would occur is easily estimated by applying the relationship between the magnetic field strength, electric current, and magnetic permeability. The relationship to $r$ (for the tubular geometry in which it occurs) is:

$$
\mathrm{r}=\mu \cdot \mathrm{I} \cdot \mathrm{B}^{-1}
$$

where $\mu=4 \pi \cdot 10^{-7} \mathrm{~N} \cdot \mathrm{A}^{-2}$, a single unit charge (for a proton) is $1.6 \cdot 10^{-19} \mathrm{~A} \cdot \mathrm{s}$, and $\mathrm{B}=1.06 \cdot 10^{7} \mathrm{~T}$ which was the calculated solution for the magnetic field strength for the relationship between the density of water and the permittivity constant.

Both unit charge and $\mu$ are constants. Assuming B is a constant, then the resulting " $r$ " should be a constant. The solution is within about $12 \%$ of $8 \pi$. Recently we have shown that the relationship between the impedance and capacitance of the universe as a single unit results in Planck's time and $8 \pi$ as an implicit geometry that represents the numbers of "turns" or a process that shares variance with inductance [12].

\section{Emergence of Velocities of Light and Excess Correlation with Mass Density}

The Alfven waves associated with magnetohydrodynamic theory have discrete velocities of propagation within the medium. The velocity can be described by:

$$
\mathbf{v}_{\mathrm{a}}=\mathrm{B}\left[\sqrt{ }\left(\mu_{\mathrm{o}} \cdot \sigma\right)\right]^{-1}
$$

If we assume this relationship is more generalized then the estimated velocity for the magnetic field strength (13.7 nT) derived from equation (1) for the average mass density of the universe and the magnetic field strength $\left(1.06 \cdot 10^{7} \mathrm{~T}\right)$ derived from (3) for the typical mass density for water, two potentially relevant velocities emerge.

For the magnetic field strength (13.7 nT) from equation (1) associated with the assumption of 1 proton per cubic meter as the average mass density of the universe, which would be very approximate to the current estimates of its total mass, the velocity would be $2.98 \cdot 10^{8} \mathrm{~m} \cdot \mathrm{s}^{-1}$ which is within measurement error of the velocity of light in a vacuum. The values from assumptions of the density for liquid water, ice, or the density of a star $\left(1.4 \cdot 10^{3} \mathrm{~kg}\right)$ are also within the range of $2.9 \cdot 10^{8} \mathrm{~m} \cdot \mathrm{s}^{-1}$.

On the other hand if the energy density of the universe remains in the denominator but the strength of the magnetic field is that derived from the density of water, the velocity from equation (4) is $2.3 \cdot 10^{23} \mathrm{~m} \cdot \mathrm{s}^{-1}$. This is the hypothesized velocity involved with the display for excess correlation entanglement that was derived by Persinger and Koren [13] by setting the product of the geometries for the three spatial dimensions and one temporal dimension of a closed surface (a circle) which was $21.3 \pi^{4} \mathrm{~m}^{7} \cdot \mathrm{s}^{-1}$ equal to the most feasible equivalent by dimensional analyses. This resulted in: 


$$
21.3 \pi^{4} \mathrm{r}^{7} \cdot \mathrm{s}^{-1}=\mathrm{G}^{2} \cdot \mathrm{M}^{2} \cdot \mathrm{d} \cdot \mathrm{t}^{3}
$$

where $\mathrm{G}$ is the Newton Gravitational constant, $\mathrm{M}$ is the mass of the universe, $d$ is its diameter and $t$ is the duration.

This order of magnitude, $10^{23} \mathrm{~m} \cdot \mathrm{s}^{-1}$, was also obtained by the ratio of the magnetic field strength associated with the total energy of the universe and its electric field strength per $\mathrm{m}$ [5]. With those assumptions the value was $\sim 10^{23} \mathrm{~m} \cdot \mathrm{s}^{-1}$. Even from a quantum perspective, the "jiffy" which is the time required for a photon moving at $\mathrm{c}$ to traverse the width of an electron $\left(\sim 10^{-23} \mathrm{~s}\right)$ converges if it is divided into the circumference of the most frequent standing width in the universe, the neutral hydrogen line $(21 \mathrm{~cm})$.

\section{Implications}

The emergence of the square of magnetic field flux intensity from the ratio of the mass density to the electric field permittivity of free space reiterates the interconnection between the electric and magnetic properties of space and the phenomenon measured as mass. The emergence of a value very proximal to the velocity of light in a vacuum when this quantification was employed in a magnetohydrodynamic context suggests that an average mass density of 1 proton per cubic meter for the universe, the permittivity of a vacuum, the permeability of a vacuum, and an interconnecting magnetic field strength may share a common source of variance that might define the whole universe as a singular set.

Although gravitational energies were not apparent, it may not be spurious that the solution between 13-14 $\mathrm{nT}$ includes the range of interplanetary magnetic field strength that is associated with the variation in Newton's Gravitational constant as measured by two different researchers over a period of 30 years [14-16]. This magnetic flux density is also approximately half of the magnetic field strength (B) for the entire universe based upon the total energy estimate of $\sim 2.2 \cdot 10^{69} \mathrm{~J}$ and when applied through a volume of $8.4 \cdot 10^{78}$ $\mathrm{m}^{3}$ by the relationship:

$$
\mathrm{B}=\sqrt{ }\left[(\mathrm{E} \cdot 2 \mu) \cdot \mathrm{m}^{-3}\right]
$$

From the context of excess correlations or non-local effects between contained volumes of masses the second solution which produces the value of $\sim 2 \cdot 10^{23} \mathrm{~m} \cdot \mathrm{s}^{-1}$ is revealing. Because this value emerged from the employment of the estimated magnetic field strength based upon the density of water but immersed within the medium of one proton per cubic meter, one interpretation is that entanglement velocity occurs primarily for specific densities of mass because they are intercalated with the spatial fabric of the universe. This is reflected in the proton density for this total set. The value of $1.67 \cdot 10^{-27} \mathrm{~kg} \cdot \mathrm{m}^{-3}$ per proton unit when multiplied by Eddington's number of $126 \cdot 2^{256}$ or $1.46 \cdot 10^{79}$ units within the universe results in a mass of $\sim 2.4 \cdot 10^{52} \mathrm{~kg}$ which is a median value for from both theoretical calculations and estimates based upon total numbers of stars each with a solar mass for the total mass of the universe.
There are two convergences that may be relevant for consideration of the dynamics by which the large-scale structure of the universe is integrated. If the excess correlation or latency to entanglement is in the order of $\sim 2 \cdot 10^{23} \mathrm{~m} \cdot \mathrm{s}^{-1}$ the time required to traverse the known functional expansion of the universe would be about $8 \mathrm{~min}$. This is not a trivial value. Within earth-bound laboratory experiments this is the duration for which demonstrations of excess correlation of photon emissions or reciprocal shifts in $\mathrm{pH}$ within discrete quantities of water between to non-local spaces that share exposure to similar rotating magnetic fields changing angular velocities and dissociated group and phase velocities occurs $[17,18]$. The excess correlation is not discernable after this duration.

It may not be adventitious that the time required for a process to occur between the earth and the sun employing the excess correlation velocity is about $10^{-12} \mathrm{~s}$ [19]. This is the typical duration of the hydronium ion before the extra proton is displaced to the next water molecule [10]. Considering the presence of water on the solar surface [20], primarily within the localized solar vortices where lower temperature prevail (sunspots) the potential for excess correlation between terrestrial and solar sources could allow a different perspective for the many "correlations" between solar activity and both chemical and biological reactions that depend upon the dynamics of water [21-25]. Such a relationship could reclassify these phenomena from eccentric chemical anomalies or observation artifacts to predictable properties of some aqueous solutions when Schrodinger-type entanglement occurs.

Although microcosm is not necessarily required to reflect macrocosm, what is measured or perceived at smaller spacetime levels is often apparent at the larger space-time levels. The duration of about 8 min or $4.8 \cdot 10^{2} \mathrm{~s}$ when divided by the total age of the universe at the final epoch, which is $3 \cdot 10^{18} \mathrm{~s}$, results in a ratio of $1.6 \cdot 10^{-16}$. If an example of quantum phenomena that has been demonstrated to occur at macroscopic levels is applied, convergence should occur. For example the Aharanov-Bohm effect [26] indicates that a phase shift occurs in an electron from an external magnetic field even though the electron beams are shielded from its influence. The typical description of this effect is:

$$
\Delta \theta=\mathrm{qVt} \cdot \hbar^{-1}
$$

where phase shift is $\Delta \theta, \mathrm{q}$ is the unit charge, $\mathrm{V}$ is the voltage, $t$ is the duration within the voltage, and $\hbar$ is the modified Planck's constant. Because $\mathrm{qV}$ is energy, the intrinsic energy associated with a phase shift of $1.6 \cdot 10^{-16}$ would be:

$$
\mathrm{E}=(\hbar \cdot \Delta \theta) \mathrm{t}
$$

Consequently the energy associated with this phase shift, which is the ratio between the time required to traverse the universe if the excess correlation velocity is valid and the age of the universe at its final epoch, would be effectively Planck's modified constant. The congruence suggests that the quantity of energy related to Planck's value is a 
manifestation of the phase shift between the duration required to traverse universal distances for the "entanglement" velocity and the total duration of the universe. If this is universal, the orbital time of an electron around a nucleus (Bohr atom) then becomes the phase shift of the standing wave for the latency to traverse the universe with respect to its total age.

Because Planck's constant is effectively the energy equivalence of the mass of an electron moving at the square of the fine structure velocity for one orbit, this apparent identity might reflect a process by which all electron orbits (and their momentum) are coupled to the upper limit of the universe's duration. The operation of an entanglement velocity could create the conditions. These conditions could be considered potential support for Mach's principle that predicts that every portion of the universe is affected by the entire universe. This concept has recently been supported by inferences from small shifts in Hubble's parameter by Bell [27]. The concomitant low level oscillations displayed periods of $39 \mathrm{Mpc}\left(12 \cdot 10^{23} \mathrm{~m}\right)$ which might be considered a standing wave for a velocity of $\sim 2 \cdot 10^{23} \mathrm{~m} \cdot \mathrm{s}^{-1}$.

\section{Conclusion}

A convergence of operations and quantifications between the smallest increments of space and time and the larges increments of space and time could reveal recondite properties and connections that could enhance prediction at these boundaries. The solutions from considering two values for matter densities and electric permittivity of space resulted in magnetic field strengths corresponding to the upper boundaries for the rest mass for photons and the quantum energy involved with displacement of protons according to Bohr's original conception. The insertion of these two magnetic intensity values into magnetohydrodynamic equations resulted in values that are very proximal to the velocity of light associated with local effects and a much larger velocity associated with non local effects that could accommodate the unusual properties of large fractional sections of space that approach the dimensions of the universe. The extraction of this velocity would suggest that Schrödinger's entanglement (excess correlation at any distance) is not instantaneous but requires a discrete latency that is potentially measurable. One consequent solution is a phase shift that relates this velocity to the specific orbital time of electrons which could intercalate all matter. These properties might be considered alternative supports for Mach's Principle and may reflect one of the major foundations of physics.

\section{References}

[1] Y. Hoffman, O. Lahav, G. Yepes, Y. Dover, "The future of the local large scale structure: the roles of dark matter and dark energy," Journal of Cosmology and Astroparticle Physics, doi: 10.1088/1475-7416/10/16, 2007.
[2] M. A. Persinger, "Convergent calculations that dark solutions are reflective of mass-energy yet to occur," International Journal of Astronomy and Astrophysics. Vol. 2, pp. 12-128, 2012.

[3] D. Hutsemekers, L. Braibant, V. Pelgrims, D. Sluse, "Alignment of quasar polarizations with large-scale structures," Astronomy \& Astrophysics, no.572, A18, 2014.

[4] D. Ryu, H. Kang and P. L. Biermann, "Cosmic magnetic fields in larges cale filaments and sheets," Astronomy and Astrophysics, Vol. 335, pp. 19-25, 1998.

[5] M. A. Persinger, "Discrepancies between predicted and observed intergalactic magnetic field strengths from the universe's total energy: it is contained within submatter spatial geometry? International Letters of Chemistry, Physics and Astronomy, Vol. 11, pp. 18-23, 2014.

[6] E. Fischbach, H. Kloor, R. A. Langel, A. T. Lui, M. Peredo, "New geomagnetic limits on the photon mass and on long range forces co-existing with electromagnetism", Physics Review Letters, Vol. 73, pp. 514-517, 1994.

[7] L. Davis, A. S. Goldharber, M. Nieto, "Jovian magnetic fields," Physics Reviews Letters, Vol. 35, pp. 1402-1405, 1975.

[8] L.-C. Tu, J. Luo, G. T. Gilles, "Limit on the photon mass deduced from Pioneer-10 observations of Jupiter's magnetic field," Reports on Progress in Physics, Vol. 68, pp. 77-130, 2005.

[9] W. C. McCLewis, A System of Physical Chemistry, Logmans, Green and Co. Bombay, 1921, pp. 115-117.

[10] T. E. DeCoursey, "Voltage-gated proton channels and other proton transfer pathways," Physiology Reviews, Vol. 83(2), pp. 475-579, 2003.

[11] M. A. Persinger, "Quantitative convergence between physicalchemical constants of the proton and the properties of water: implications for sequestered magnetic field and a universal quantity," International Letters of Chemistry, Physics and Astronomy, Vol. 2, pp. 1-10, 2014.

[12] M. A. Persinger, S. A. Koren, "The product of the calculated impedance and capacitance of the universe solves for Planck's time and $8 \pi$," Journal of Advances in Physics, Vol. 11, pp. 2347-3487, 2016.

[13] M. A. Persinger, S. A. Koren, "Dimensional analyses of geometric products and boundary conditions of the universe: implications for a quantitative value for the latency to display entanglement," The Open Astronomy Journal, Vol. 6, pp. 110-13, 2013.

[14] B. M. Vladmirsky, A. V. Bruns, "Influence of the sector structure of the interplanetary magnetic field on the results of measurements of the gravitational constant," Biophysics, Vol. 43, pp. 720-725, 1998.

[15] T. Quinn, H. Parks, C. Speake, R. Davis, "Improved determination of G using two method," Physics Letters, 111, 101102, 2013.

[16] M. A. Persinger, L. S. St-Pierre, "Is there a geomagnetic component involved with the determination of G?" International Journal of Geosciences, Vol. 5, pp. 450-452, 2014. 
[17] B. T. Dotta, M. A. Persinger, "Doubling"” of local photon emissions when two simultaneous spatially separated, chemiluminescent reactions share the same magnetic field configurations," Journal of Biophysical Chemistry, Vol. 3, pp. 72-80, 2012.

[18] B. T. Dotta, N. J. Murugan, L. M. Karbowski, M. A. Persinger, "Excessive correlated shifts in $\mathrm{pH}$ with distal solutions sharing phase-uncoupled angular accelerating magnetic fields: macroentanglement and information transfer," International Journal of Physical Sciences, Vol. 8, pp. 1783-1787, 2013.

[19] M. A. Persinger, S. A. Koren, "Potential role of the entanglement velocity of $10^{23} \mathrm{~m} \cdot \mathrm{s}^{-1}$ to accommodate recent measurements of large scale structures of the universe. International Letters of Chemistry, Physics and Astronomy, Vol. 3, pp. 106-112, 2015.

[20] T. Oka, "Water on the Sun: molecules everywhere. Science, Vol. 277, pp. 328-329, 1997.

[21] N. V. Klocheck, L. E. Palamarchuk, M. V. Nikonova, "Preliminary results of investigations into the effect of cosmophysical radiation of a non-electromagnetic nature on physical and biological systems," Biophysics, Vol. 40, pp. 883-891, 1995.
[22] G. Piccardi, The Chemical Bases of Medical Climatology, C. C. Thomas, Ill., 1967.

[23] M. Takata. Uber eine neue biologisch wirksame Komponente der Sonnenstrahlug. Archives fur Meteorologie, Geophysik und Bioklimatologie, Vol. 2, 486-508, 1951.

[24] T. A. Moraes, P. W. Barlow, E. Kingele, C M. Gallep, Spontaneous ultra-weak light emissions from wheat seedlings are rhythmic and synchronized with the time profile of the local gravimetric tide. Naturwissenschafen, Vol. 99, 465-472, 2012.

[25] L. Y. Berzhanskaya, B. V. Berzhanskii, O. Y. Beloptovota, T. G. Pil'nnikova, T. N. Metlyayev. Bacterial bioluminescent activity as a pointer to geomagnetic disturbances. Biophysics, Vol. 40, 761-764, 1995.

[26] A. Tonomura, N. Osakabe, T. Matsuda, T, Kawaski, J. Endo, "Evidence of Aharanov-Bohm effect with magnetic field completely shielded from electron wave," Physics Review Letters, Vol. 56, pp. 792-795, 1986.

[27] M. B. Bell, "Mach's principle of inertia is supported by recent astronomical evidence," International Journal of Astronomy and Astrophysics, Vol. 5, pp. 166-172, 2015. 